\title{
Crystal structure of ethyl (Z)-2-hydroxyimino-2-(methylsulfanyl)acetate, $\mathrm{C}_{5} \mathrm{H}_{9} \mathrm{SNO}_{3}$
}

\author{
A. Cousson ${ }^{1}$ and J.-M. Coustard ${ }^{*, 11}$ \\ I Laboratoire Leon Brillouin (CEA-CNRS), CE Saclay, F-91191 Gif-sur-Yvette Cedex, France \\ II Universite de Poitiers, Faculte des Sciences, Laboratoire de Chimie 12. UMR-CNRS 6514, 40, avenue du recteur Pineau, F-86022 Poitiers Cedex, France
}

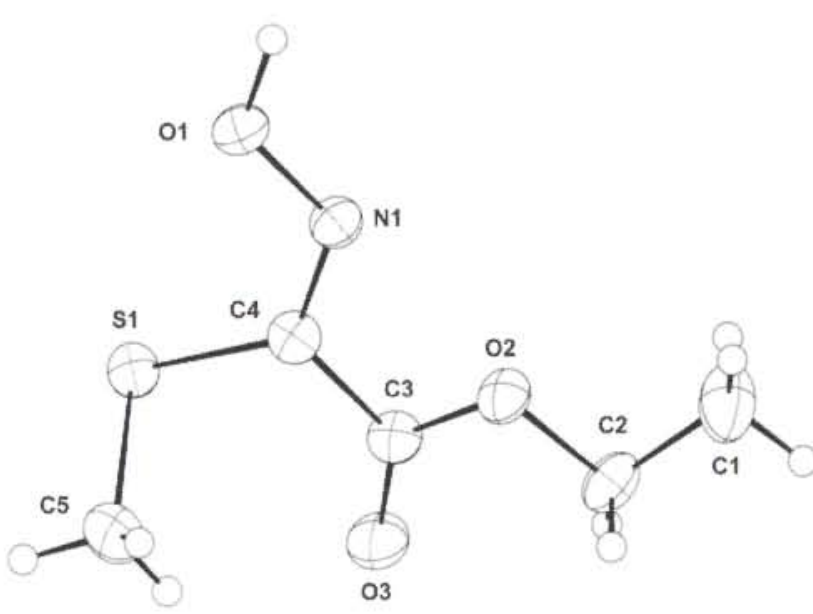

\begin{abstract}
$\mathrm{C}_{5} \mathrm{H}_{9} \mathrm{NO}_{3} \mathrm{~S}$, triclinic, $P \overline{\mathrm{I}}$ (No. 2), $a=5.278(3) \AA, b=8.235(4) \AA$, $c=9.667(2) \AA, \alpha=95.26(4)^{\circ}, \beta=103.68(4)^{\circ}, \gamma=102.16(4)^{\circ}$, $V=394.6 \AA^{3}, Z=2, R_{\mathrm{gt}}(F)=0.066, w R(F)=0.079, T=293 \mathrm{~K}$.
\end{abstract}

\section{Source of material}

Ethyl nitroacetate was dissolved in triflic acid at $273 \mathrm{~K}-278 \mathrm{~K}$. After about 30 minute reaction time, the solution was quenched by a mixture of methanethiol and $\mathrm{CH}_{2} \mathrm{Cl}_{2}$ at $193 \mathrm{~K}-213 \mathrm{~K}$. Extraction with $\mathrm{CH}_{2} \mathrm{Cl}_{2}$ and flash-chromatography afforded the title compound [1]. The compound was crystallized from dichloromethane/petroleum ether.

\section{Discussion}

Ethyl nitroacetate is readily dissolved in triflic acid, sulfuric acid or $\mathrm{HF}_{-} \mathrm{SbF}_{5}$. In these very acidic media, ethyl nitroacetate is doubly protonated and form a conjugated hydroxynitrilium ion that can be trapped by aromatics [2] or $\mathrm{MeSH}$ to affords the title compound [1]. The present X-ray analysis of the title compound shows that the molecule has a nearly planar conformation, with a maximum deviation from the best plane of $0.554 \AA$. The torsion angle $\mathrm{C}-\mathrm{S}-\mathrm{C}=\mathrm{N}$, with a value of $\tau_{1}=-157.94^{\circ}$, indicates a s-cis conformation for the $-\mathrm{S}-\mathrm{C}=$ single bond. The conjugated bonds $\mathrm{N}=\mathrm{C}-\mathrm{C}=\mathrm{O}$ are nearly coplanar $\left(\tau_{2}=-157.86^{\circ}\right)$, which allows delocalization of the $\pi$-electronic system, affording a better electronic stabilization. The planar $\mathrm{S}-\mathrm{C}=\mathrm{N}-\mathrm{O}(\mathrm{H})$ system $\left(\tau_{3}=1.59^{\circ}\right.$ value) is $Z$ configured with $\mathrm{MeS}$ and $\mathrm{N}-\mathrm{OH}$ in the cis geometrical position. This last feature is in agreement with theoretical considerations concerning nucleophilic addition on triple bond $[3,4]$ and, in the present case, of MeSH on the $\mathrm{CN}$ triple bond of the hydroxynitrilium ion formed from ethyl nitroacetate.

Table 1. Data collection and handling.
Crystal: Wavelength: $\mu$ : Diffractometer, scan mode: $20_{\text {max }}$ : $N(h k l)_{\text {measured, }} N(h k l)_{\text {unique: }}$ Criterion for $\left.I_{\mathrm{obs}}, N(h k l)\right)_{\mathrm{gl}}$ : $N(\text { param })_{\text {refined: }}$ Programs: colorless prism, size $0.15 \times 0.20 \times 0.25 \mathrm{~mm}$ Mo $K_{\alpha}$ radiation $(0.71073 \AA)$ $34.5 \mathrm{~cm}^{-1}$

Enraf-Nonius CAD4, $\omega / 2 \theta$ $50^{\circ}$ 2776, 925

$I_{\mathrm{obs}}>3 \sigma\left(I_{\mathrm{obs}}\right), 925$

94

SHELXS-86 [5], CRYSTALS [6], CAMERON [7]
Table 2. Atomic coordinates and displacement parameters (in $\AA^{2}$ ).

\begin{tabular}{llrrrl}
\hline Atom & Site & \multicolumn{1}{c}{$x$} & \multicolumn{1}{l}{$y$} & \multicolumn{1}{l}{$z$} & \multicolumn{1}{l}{$U_{\text {iso }}$} \\
\hline $\mathrm{H}(1)$ & $2 i$ & -0.2625 & -0.0567 & 0.3825 & $0.172(8)$ \\
$\mathrm{H}(11)$ & $2 i$ & 0.9856 & 0.3952 & 0.861 & $0.131(8)$ \\
$\mathrm{H}(12)$ & $2 i$ & 0.6579 & 0.3547 & 0.8134 & $0.131(8)$ \\
$\mathrm{H}(13)$ & $2 i$ & 0.8099 & 0.2023 & 0.8032 & $0.131(8)$ \\
$\mathrm{H}(21)$ & $2 i$ & 0.8332 & 0.4672 & 0.6327 & $0.109(8)$ \\
$\mathrm{H}(22)$ & $2 i$ & 0.9852 & 0.3149 & 0.6225 & $0.109(8)$ \\
$\mathrm{H}(51)$ & $2 i$ & 0.3608 & 0.2164 & -0.0263 & $0.107(8)$ \\
$\mathrm{H}(52)$ & $2 i$ & 0.5307 & 0.3298 & 0.1283 & $0.107(8)$ \\
$\mathrm{H}(53)$ & $2 i$ & 0.5227 & 0.1299 & 0.1008 & $0.107(8)$ \\
\hline
\end{tabular}


Table 3. Atomic coordinates and displacement parameters (in $\AA^{2}$ ).

\begin{tabular}{|c|c|c|c|c|c|c|c|c|c|c|}
\hline Atom & Site & $x$ & $y$ & $z$ & $U_{11}$ & $U_{22}$ & $U_{33}$ & $U_{12}$ & $U_{13}$ & $U_{23}$ \\
\hline$S(1)$ & $2 i$ & $0.1246(2)$ & $0.1687(2)$ & $0.1469(1)$ & $0.0534(7)$ & $0.0761(9)$ & $0.0507(7)$ & $0.0108(6)$ & $0.0131(5)$ & $0.0088(5)$ \\
\hline$C(1)$ & $2 i$ & $0.817(1)$ & $0.3228(8)$ & $0.7917(6)$ & $0.108(5)$ & $0.071(4)$ & $0.063(3)$ & $0.013(3)$ & $0.000(3)$ & $-0.001(3)$ \\
\hline $\mathrm{C}(2)$ & $2 i$ & $0.828(1)$ & $0.3466(6)$ & $0.6435(5)$ & $0.051(3)$ & $0.043(2)$ & $0.068(3)$ & $0.004(2)$ & $0.002(2)$ & $-0.004(2)$ \\
\hline $\mathrm{C}(3)$ & $2 i$ & $0.5087(9)$ & $0.2836(5)$ & $0.4169(5)$ & $0.054(2)$ & $0.028(2)$ & $0.056(2)$ & $0.011(2)$ & $0.016(2)$ & $0.004(2)$ \\
\hline $\mathrm{C}(4)$ & $2 i$ & $0.2463(8)$ & $0.1747(5)$ & $0.3311(4)$ & $0.053(2)$ & $0.026(2)$ & $0.051(2)$ & $0.014(2)$ & $0.016(2)$ & $0.004(2)$ \\
\hline $\mathrm{C}(5)$ & $2 i$ & $0.415(1)$ & $0.2178(8)$ & $0.0806(6)$ & $0.070(3)$ & $0.104(5)$ & $0.061(3)$ & $0.018(3)$ & $0.029(3)$ & $0.009(3)$ \\
\hline$N(1)$ & $2 i$ & $0.1031(7)$ & $0.0974(4)$ & $0.4058(4)$ & $0.052(2)$ & $0.036(2)$ & $0.051(2)$ & $0.005(1)$ & $0.012(2)$ & $0.002(1)$ \\
\hline$O(1)$ & $2 i$ & $-0.1504(6)$ & $0.0071(4)$ & $0.3257(3)$ & $0.051(2)$ & $0.051(2)$ & $0.059(2)$ & $-0.002(1)$ & $0.012(1)$ & $0.007(1)$ \\
\hline$O(2)$ & $2 i$ & $0.5837(6)$ & $0.2410(3)$ & $0.5456(3)$ & $0.057(2)$ & $0.035(2)$ & $0.055(2)$ & $0.003(1)$ & $0.007(1)$ & $0.004(1)$ \\
\hline$O(3)$ & $2 i$ & $0.6322(8)$ & $0.3986(4)$ & $0.3744(4)$ & $0.073(2)$ & $0.055(2)$ & $0.075(2)$ & $-0.009(2)$ & $0.012(2)$ & $0.017(2)$ \\
\hline
\end{tabular}

\section{References}

1. Coustard, J. M.: 1,1-bis(Methylthio)-2-Nitroethene in Superacids: NMR study and Reactivity of the Formed Hydroxynitrilium Ion. Tetrahedron 51 (1995) 10929-10940.

2. Coustard, J. M.; Jacquesy, J. C.; Violeau, B.: Hydroxynitrilium Ions as Intermediates in the Reaction of Nitroderivatives with Aromatics. Tetrahedron Lett. 32 (1991) 3075-3078.

3. Hegarty, A. F.: Stereospecific Reactions of Nitrilium Ions and Analogous 1,3-Dipoles. Acc. Chem. Res. 13 (1980) 448-454.
4. Nguyen, M. T.; Malone, S., Hegarty, A. F.; Williams, I.I.: Nucleophilic Additions to Nitrile Oxides: concerted or stepwise? J. Org. Chem. 56 (1991) 3683-3687.

5. Sheldrick, G. M.: SHELXS86, Program for Crystal Structure Determination. University of Göttingen, Germany, 1986.

6. Watkin, D. J; Carruthers, J. R.; Betteridge, P. W.: CRYSTALS User Guide, Chemical Crystallography Laboratory, University of Oxford, England, 1994.

7. Pearce, L.; Watkin, D. J.; Prout, C. K.: CAMERON. Program for Plotting Molecular Structures. Chemical Crystallography Laboratory, University of Oxford, England, 1994. 